\title{
Antepartum and intrapartum cardiotocography and fetal outcome in high-risk pregnancy
}

\author{
I Bina ${ }^{1}$, T Parveen$^{2}$, A Khanom ${ }^{3}$, MD Akhter ${ }^{4}$
}

\begin{abstract}
The objective of the study was to find out the incidence of abnormal cardiotocography (CTG) findings in pregnancies at risk, to find out the suitable time of delivery of the fetus at risk and to find out the fetal outcome in abnormal antepartum CTG findings. It is a prospective study where 450 highrisk pregnancy cases of different gestational age, ranging from 32-42 weeks were selected from BSMMU, Dhaka and Khalishpur Clinic, Khulna from 2003 to 2013. CTG was done on admission and the subjects were followed-up till delivery. The data obtained from the present study were compiled and appropriate statistical analysis was carried out using SPSS. Out of the cases, normal CTG was found in 357 cases and abnormal CTG was found in 93 cases. Among these 93 cases, normal outcome was found in 87 cases (93.1\%) and abnormal outcome in 6 cases (6.9\%). The specificity, sensitivity, negative predictive value and positive predictive value for antepartum CTG was $92.5 \%, 20.5 \%, 81.9 \%$ and $49.3 \%$, respectively. This study revealed that CTG shows more accuracy in case of healthy fetus that a normal CTG almost always gave a normal fetal outcome. But if CTG showed abnormality, the fetal outcome may be normal. So a more accurate correlation with other biophysical and biochemical parameters of fetus is to be done for further evaluation.
\end{abstract}

Key words: Antepartum, intrapartum, cardiotocography, fetal outcome, highrisk pregnancy

\section{Introduction}

Obstetrics have long been looked for antenatal test that would identify the fetus at risk of intrauterine hypoxia and death. Ideally such a test should not only be reliable but performed easily and repeatedly. The results should be available immediately and the cost should be minimum. ${ }^{1}$

Use of fetal heart rate (FHR) to assess fetal well-being has become standard obstetric practice. $^{2}$ Currently the most commonly

1. Dr. Ismatara Bina MD, DGO, MCPS, FCPS(Obs \& Gynae), MRCOG, Consultant Obstetrician \& Gynaecologist,

Khalishpur Clinic, House- 41, Rd- 101, Khulna- 9000. Tel: +88-041761637.

2. Dr. Tabassum Parveen MBBS, FCPS(Obs \& Gynae)

3. Dr. Afroza Khanom MBBS, MS(Obs \& Gynae)

4. Dr. Ms. Dalia Akhter MBBS, FCPS(Obs \& Gynae) 
hypoxia. ${ }^{3}$ The fetal heart rate signal can be obtained from a fetal ECG electrode placed on the maternal abdomen by processing the Doppler shift of an ultrasound beam. ${ }^{4}$ Prenatal CTG is aimed to detect the effect of acute and/or chronic fetal hypoxia. The goal of the obstetrician/perinatologist caring for the pregnant women is not only to prevent fetal death but with the detection of fetal compromise and timely delivery of such infants in an effort to maximize their future potential. ${ }^{5}$ A large number of antepartum fetal deaths are caused by uteroplacental insufficiency. Antepartum fetal testing by identifying the uteroplacental insufficiency and timely action has prevented many fetal deaths. ${ }^{6}$ Similarly intrapartum fetal monitoring by detecting fetal distress in early stages has saved many fetus from demise. ${ }^{7}$

CTG was first developed in 1950 and became commercially available in $1960 .^{3}$ CTG was first used in labour to detect fetal distress but later introduced in antepartum fetal surveillance as contraction stress test on the same principles. The aim of the study was to find out the incidence of abnormal CTG in pregnancies at risk, to find out the suitable time of delivery of the fetus at risk and to find out the fetal outcome in women with abnormal antepartum CTG findings.

\section{Methods}

It is a prospective study where 450 high-risk pregnancy cases of different gestational age, ranging from 32-42 weeks were randomly selected from BSMMU, Dhaka and Khalishpur Clinic, Khulna from 2003 to 2013. CTG was done on admission and the subjects were followed-up till delivery. The data obtained from the present study were compiled and appropriate statistical analysis was carried out using SPSS.

\section{Inclusion criteria}

Pregnant women between 32-42 weeks of gestation and with the following indications of high-risk pregnancy were included in this study: post-dated pregnancy, pregnancyinduced hypertension, bad obstetric history (history of stillbirth, intrauterine death), intrauterine growth retardation, decreased fetal movement, pregnancy with pervious history of caesarean delivery, pregnancy with maternal disease like diabetes mellitus, heart disease, systemic lupus erythematosus, chronic nephritis, antepartum haemorrhage (abruption placenta, placenta praevia), Rhisoimmunization, oligohydramnios, pre-mature rupture of membrane, multiple pregnancy, etc.

\section{Exclusion criteria}

Pregnant women with the following criteria were excluded from this study: pregnant women with low-risk pregnancy, gestational age <32 weeks, women with high-risk pregnancy who went to labour, pregnancy with congenital fetal anomalies, etc.

\section{Method of testing}

High-risk cases were identified through history, antenatal records and clinical examination. External (from maternal abdominal wall- non invasive process) continuous tracing of FHR was obtained using ultrasound Doppler Effect. Pregnant women were placed in semi-recumbent position with slight left lateral tilt. The transducers were placed on the maternal abdomen: one over the fundus and other at a site where the fetal heart sound was best audible. Uterine contraction was recorded simultaneously by tocodynamo meter. Blood pressure was measured at the initiation of the test and at every 15 minutes thereafter.

\section{Results}

Out of 450 high-risk pregnancy cases, normal CTG was found in 357 cases and abnormal CTG was found in 93 cases. Among these 93 cases, normal outcome was found in 87 cases $(93.1 \%)$ and abnormal outcome in 6 cases (6.9\%). The specificity and sensitivity for antepartum CTG was $92.5 \%$ and $20.5 \%$, respectively (Table 1 ). Negative predictive value and positive predictive value for antepartum CTG was $81.9 \%$ and $49.3 \%$, respectively. There was significant difference between abnormal and normal tests results in predicting over all abnormal fetal outcomes.

\section{Discussion}

Antepartum fetal surveillance is used to 
Table 1. Performance of characteristics of antepartum cardiotocogram for each of the different fetal outcome

\begin{tabular}{lcc}
\hline Outcome & Sensitivity (\%) & Specificity (\%) \\
\hline Overall abnormal outcome & 20.5 & 92.5 \\
Low 1-minute Apgar score & 43.5 & 79.8 \\
Low 5-minute Apgar score & 9.7 & 98.3 \\
Small for gestation & 19.4 & 93.3 \\
Admission in Neonatal Intensive Care Unit & 9.7 & 98.3 \\
\hline
\end{tabular}

identify potential fetal jeopardy. Although both antepartum CTG and biophysical profile are used in office or hospital settings and opinion differs as to which test is preferable. The rate of fetal demise without CTG in highrisk pregnancies is $10-30$ per 1000 with antepartum surveillance only, this rate is nil in our study population. There was no neonatal or perinatal death. Even in unmonitored low-risk pregnancies the rate of fetal demises is 2-4 per 1000 live birth. ${ }^{8}$ The present study was carried out to determine the incidence of abnormal antepartum cardiotocogram, thereby correlating its sensitivity and specificity. Prognostic value of the test was assessed in terms of the incidence of abnormal outcome of fetus. The study also showed the positive predictive value of the test, which indicates its reliability as a diagnostic test.

In the present study, in most cases, delivery occurred within 3-4 days of the last test. Evaluation of abnormal test in respect to overall abnormal pregnancy outcome was done. Abnormal tests are more predictor of abnormal outcome than normal tests, which is similar to the observation of Platt et al. ${ }^{9}$ Specific outcome, i.e. low 1-minute Apgar score, low 5-minute Apgar score, small for gestational age fetus and admission into neonatal intensive care unit. The reason for higher incidence of abnormal test seems to be due to inclusion of high-risk cases with risk of intrauterine hypoxia and because of strict standard maintained to include pregnant women in the study sample. There might be also intra-observer and inter- observer variation in interpretation of test results.

In this study, sensitivity and specificity was $20.5 \%$ and $92.5 \%$, respectively. These are comparable to other study. ${ }^{10}$ It means that computerized CTG shows more accuracy in case of healthy fetus than a normal CTG almost always gave a normal fetal outcome. But if CTG showed abnormal, the fetal outcome may be normal. So a more accurate correlation with other biophysical and biochemical parameters of fetus is to be done for further evaluation. In a study Bari et al reported that post-natal mortality rate was 151 per 1000 total birth. ${ }^{11}$ It indicates that computerized CTG improves accuracy and allows distinguishing fetuses that are truly jeopardized because of hypoxemia, from those who are not. So as our hospital is a tertiary as well as teaching center with good neonatal backup, timely intervention dictated by antepartum cardiotocogram results such a neonatal outcome.

\section{Conclusion}

Out of the study sample 450 high-risk pregnancy cases of different gestational age, ranging from 32-42 weeks, normal CTG was found in 357 cases and abnormal CTG was found in 93 cases. Among these 93 cases, normal outcome was found in 87 cases $(93.1 \%)$ and abnormal outcome in 6 cases $(6.9 \%)$. The specificity, sensitivity, negative predictive value and positive predictive value for antepartum CTG was 92.5\%, 20.5\%, $81.9 \%$ and $49.3 \%$, respectively. This study revealed that CTG shows more accuracy in 
case of healthy fetus that a normal CTG almost always gave a normal fetal outcome. But if CTG showed abnormality, the fetal outcome may be normal. So, it is needed to be careful in dealing with abnormal CTG, as these babies need further evaluation before final decision about their mode of delivery.

\section{References}

1. Patel NB, Owen P. Fetal Assessment in the third trimester: biophysical methods. In: Chemberline G, editor. Turnbull's obstetrics. 2nd ed. Edinburgh: Churchill Livingstone, 1995:231-52.

2. Spencer JA. Cardiotocographic assessment of fetal wellbeing in late pregnancy and labour. In: Recent Advances in Obtetrics and Gynaecology. No 21. Edinburgh: Churchill Livingstone, 2001:1-20.

3. Parer JT and King T. Fetal heart rate monitoring: Is it salvageable? Am J Obstet Gynecol 2000;182:982-7.

4. Spencer JA. Current methods of continuous FHR monitoring. Prof Nurses 1992;8(3):173-5.

5. Gabbe SG, Niebyl JR, Simpson JL. Obstetrics: normal and problem pregnancies: 3rd edition. New York:Churchill Livingstone, 1996:327329,339,403-4.
6. Schneider EP, Hutson JM, Petric RH. An assessment of the first decade's experience with antepartum foetal heart rate testing. Am J Perinatol 1998;5(2):134-41.

7. Yeh SY, Diaz F, Paul RH. Ten-year experience of intrapartum foetal monitoring in Los Angeles County/ University of Southern California Medical Centre. Am J Obstet Gynecol 1982;143(5):496-500.

8. Sokol RJ, Jones TB, Pernoll ML. Methods of assessment for pregnancy at risk. In: Decherney $\mathrm{AH}$, Pernoll ML. Current Obstetrics and Gynecologic Diagnosis and Treatment. New Jersey: Prentice-Hall International, Inc., 1994:275-305.

9. Platt LD, Walla $C A$, Paul $R H$, et al. A prospective trial of the fetal biophysical profile versus the nonstress test in the management of high-risk pregnancies. Am J Obstet Gynecol 1985;153(6):624-33.

10. Kierse MJ and Trimbos JB. Assessment of antepartum cardiotocogramm in of high-risk pregnancy. Br J Obstet Gynecol 1980;87(4):261-9.

11. Bari I, Siddiquey AHM, Rahman MS, Hossain MA. Perinatal mortality in a teaching hospital. TAJ (Rajshahi) 1996;9:95-7. 